\section{Implementing growth monitoring recommendations}

The Canadian Task Force on Preventive Health Care recommends growth monitoring for all children at all primary care visits to screen for overweight and obesity. ${ }^{1}$ Height and weight data are not only necessary for primary care providers to monitor the growth of their patients, but also may provide an excellent opportunity for public health units and researchers to assess the growth of children across Canada.

With the increased use of electronic medical records (EMRs), a new source of measured height and weight data is available. ${ }^{2}$ Given these recommendations and the potential use of these data for surveillance, we need to consider the current state of growth monitoring practices in primary care. To do this, we need to address access to appropriate equipment and techniques to measure height and weight, ensure the use of appropriate and updated growth charts integrated into EMRs, and better understand who attends primary care and any implications of monitoring growth at all visits, including well-child visits and others. These issues are important for the clinical care of children, and affect how these data are used for surveillance purposes.

The Task Force suggests standards for height and weight measurement. It is unknown whether health care providers are already following these standards.

Some practitioners may use the "paper and pencil" method, where the child lies on an examining table and the distance between a mark at the head and foot is determined with a measuring tape. However, this method has been shown to overestimate length compared to the use of a standardized length board. ${ }^{3}$ It is unclear how prevalent this practice is in Canada, and what would be needed for providers to have access to correct equipment, such as a length board, and appropriate training to use the equipment.
Canadian standards for growth monitoring have recommended the use of the WHO growth charts since 2010, further updated in 2014, which replaced the 2000 American growth charts from the Centers for Disease Control and Prevention. ${ }^{4}$ It is unknown what proportion of primary care providers are aware of this recommendation. Use of the appropriate growth charts may be limited by the availability of EMR tools or embedded into existing EMR software. Even if EMR vendors can provide appropriate and updated growth charts, who is responsible for paying for and implementing these upgrades?

Height and weight data from EMRs may be highly valuable for healthy growth surveillance for public health research. It is important to acknowledge that even with accurate equipment, measurement procedures and up-to-date growth charts, there may be variability in use during primary care visits by age or region. Although children 0 to 5 years old attend well-child visits regularly to receive vaccinations, older children and adolescents tend to visit their physicians only intermittently or when sick. Data from a previous study showed that the proportion of children who visited their primary care provider decreased from $84 \%$ at less than 1 year old to $11 \%$ for adolescents 15 to 19 years old. ${ }^{5}$

Finally, the Task Force recommends height and weight measurement at "all appropriate primary care visits," which includes both well-child and sick visits. Using all physical measurement data is then subject to possible selection bias because children with unhealthy weights may be visiting their primary care providers more than their healthy weight peers. We are encouraged by the adoption of growth monitoring for primary care providers of children; the devil is in the details for effective implementation of this important recommendation.

Sarah E. Carsley MSc, Karen Tu MD MSc, Catherine Birken MD MSc University of Toronto, Toronto, Ont.

\section{References}

1. Canadian Task Force on Preventive Health Care. Recommendations for growth monitoring, and prevention and management of overweight and obesity in children and youth in primary care. CMAJ 2015; 187:411-21

2. Birtwhistle R, Tyler W. Primary care electronic medical records: a new data source for research in Canada. CMAJ 2015; 187:239-40.

3. Rifas-Shiman SL, Gillman MW, Oken E, et al. Similarity of the CDC and WHO weight-for-length growth charts in predicting risk of obesity at age 5 years. Obesity (Silver Spring) 2012;20:1261-5.

4. Dietitians of Canada, et al. Promoting optimal monitoring of child growth in Canada: using the new WHO growth charts. Can J Diet Pract Res 2010; 71: e1-3.

5. Birken $\mathrm{C}, \mathrm{Tu} \mathrm{K}$, Oud W, et al. Overweight and obesity rates in children in Ontario, Canada using electronic medical records [abstract]. Pediatric Academic Society Annual Conference; 2014 May 3-6; Vancouver.

CMAJ 2015. DOI:10.1503/cmaj.1150073

\section{Another mini-masterpiece}

This is yet another affecting piece in the Humanities section that hit home. ${ }^{1}$

Thanks Dr. Feld and CMAJ for publishing this little story that struck many chords in me. I was instantly brought to tears. The recognizability was overwhelmingly intense. How we seem to ourselves is likely unlike what we seem to our patients, except in rare and nearly religious moments, such as the one described so well.

Keep it up please!

\section{Duncan S. Armstrong MD}

Halifax, NS

\section{Reference}

1. Feld ED: With friends like that ... . CMAJ 2015;187:917.

CMAJ 2015. DOI:10.1503/cmaj.1150075

\section{Letters to the editor}

In submitting a letter, you automatically consent to having it appear online/in print. All letters accepted for print will be edited for space and style. See www.cmaj.ca for full versions and competing interests. 